\title{
REPRODUCTIVITY AND VALIDITY ANALYSIS OF BADMINTON PLAYER COGNITIVE PERFORMANCE USING BADMINTON REACTION INHIBITION TEST (BRIT)
}

\author{
Handayani Nila Praja ${ }^{1 *}$, Rivo Panji Yudha ${ }^{2}$, Sri Sundari ${ }^{3}$, Wahyu Adhi Nugroho ${ }^{4}$ \\ ${ }^{1234}$ Faculty of Teacher Training and Education, Universitas 17 August 1945 Cirebon, West Java, \\ Indonesia
}

\begin{tabular}{l}
\hline Info Artikel \\
Article History: \\
Received September 2020 \\
Revised September 2020 \\
Accepted September 2020 \\
Available online September 2020 \\
\hline \\
Keywords: \\
Badminton, reaction time, \\
inhibition control, athletes \\
\hline
\end{tabular}

\begin{abstract}
The purpose of this study was to analyze the reproducibility and validity of the Badminton Reaction Inhibition Test (BRIT) or the Badminton Reaction Barrier Test. BRIT measures four components: general-domain reaction time, badminton-specific reaction time, general-domain inhibition control, and badminton-specific inhibition control. Fifteen male national badminton athletes and nine non-national badminton players in Region 3 Cirebon participated in this study. Five participants were retested within three weeks on a specific badminton component. Reproducibility was acceptable for badminton specific reaction times (ICC $=0.626$, $\mathrm{CV}=6 \%$ ) and for badminton specific inhibition controls (ICC = $0.317, \mathrm{CV}=13 \%$ ). The validity of a good construct was shown in the specific poor reaction time that differentiated between national level athletes and non-national athletes $(\mathrm{F}=6.650, \mathrm{p}<0.05)$. National level athletes did not outperform non-national athletes on general-domain reaction times or on both inhibitory control components ( $p>0.05$ ). Concurrent validity for general-domain reaction times was good, as it was associated with the national rankings for national level athletes ( $\rho$ pro $=0.70, \mathrm{p}<0.01$ ) and nonnational athletes $(\rho=0.70, p<0.05)$. ). No relationship was found between national ranking and badminton specific reaction time, nor between the two inhibition control components ( $p>0.05$ ). In conclusion, the reproducibility and validity of the control inhibition assessment are not confirmed, however, BRIT appears to be a valid and reproducible measure of reaction time in badminton players. Reaction times as measured by BRIT can provide input for training programs aimed at improving badminton player performance.
\end{abstract}

\begin{tabular}{ll}
\hline Corresponding address & : Jl. Perjuangan No.17, Karyamulya, Kec. Kesambi, \\
& Kota Cirebon, Jawa Barat \\
Email & $:$ handa07nila@gmail.com
\end{tabular}




\section{INTRODUCTION}

Badminton is an intercept sport, a fast racket in terms of shuttle speed with an average speed of up to $70 \mathrm{~m} / \mathrm{s}$ (Phomsoupha and Laffaye, 2014, 2015). An average of six strokes is played during a seven second rally and the mean frequency of strokes is high with approximately one stroke per second (Phomsoupha and Laffaye, 2015). To compensate for the high game speed, fast reaction times are essential to achieve pro performance (Loureiro and Freitas, 2012). Unpredictable opponent actions and the use of feints in elite badminton suggest inhibition control is also important for pro performance. Although it is recognized that a combination of excellence in technical, tactical, anthropometric, physical and mental skills is required for pro sports performance (Elferink-Gemser et al., 2011), Fast reaction times and excellent inhibition control are suggested to be indispensable for badminton expertise. However, due to the limited literature available, the importance of reaction time and inhibition control for reaching and performing at the national level in badminton can only be estimated.

Reaction time is a measure of processing speed and reflects response efficiency in information processing tasks. Fast reaction times are deemed necessary for fast and accurate reactions in fast-paced sports(Voss et al., 2010). Reaction time is considered a low-level cognitive function that supports basic information processing (Wetherell, 1997). Inhibition control refers to the ability to stop a planned or dominant motor response and is a high-order cognitive function (Williams et al., 1999). High-level cognitive functions are often called executive functions and are important in tasks that require concentration, coordination, change and the exclusion of strong internal or external attractions (Diamond, 2006).

The relationship between lowlevel cognitive performance and exercise performance is described in the reviews of Voss and Kramer (2010) and Mann et al. (2007). Voss and Kramer's (2010) review examined general-domain reaction time through a component skills approach, which measures cognitive performance in contexts outside of sport (Alves et al., 2013). Mann et al. (2007) examined context-specific reaction times and included studies that applied an expert performance approach. This approach uses a test setting that reflects the demands of a particular sport. Apart from their different approaches, both reviews suggest that fast reaction times are important for athlete performance, especially in interceptive sports such as badminton (Mann et al., 2007; Voss et al., 2010).

Recent studies that have focused on high-level cognitive performance in soccer have revealed that pro or gifted soccer players beat non-pro players on a common-domain inhibitory control task (Huijgen et al., 2015; Verburgh et al., 2014; Vestberg et al. ., 2012). Kida et al. (2005) assessed context-specific inhibitory control of baseball players and were able to distinguish not only national from nonnational players, but also from subnational players. In summary, generalized and context-specific control measures of 
inhibition seem to differentiate between players of different performance levels (Huijgen et al., 2015; Kida et al., 2005; Verburgh et al., 2014; Vestberg et al., 2012).

To date, the literature on reaction time and inhibition control in badminton players of various performance levels is limited. As an exception, Loureiro and Freitas (2012) state that badminton players at the national level have badminton specific reaction times that are much faster than non-national level players. However, this study did not provide an evaluation of the reproducibility and validity of the tests used and did not include an assessment of inhibitory controls. For accurate checks of reaction time and inhibition control in badminton players of varying performance levels, badminton-general and specificdomain reaction times and inhibition controls should be assessed. Considering that, to our knowledge, no such test exists (Faber et al., 2016), a test that measures four components is developed: general reaction time, badminton specific reaction time, general inhibition domain control, and badminton specific inhibition control. For general-domain reaction time assessment and inhibitory control, a procedure similar to the Stop Signal Task was included in the test (Williams et al., 1999).

Badminton specific reaction times and inhibition controls were assessed in a procedure based on the badminton special test used by Loureiro and Freitas (2012), complemented by the characteristics of a badminton-specific match play. The special characteristics of badminton include the movement to reach the arm to simulate stroke execution, the middle semi-squat position (Loureiro and Freitas, 2012), fast full-body movement to the front and back of the hand (i.e. lunges) (Hong et al., 2014; Kuntze et al., 2010) and visual cues (Lees, 2003; Phomsoupha and Laffaye, 2015).

\section{METHODS}

A total of 24 male badminton players Region 3 Cirebon West Java divided into two groups participated in the study. The national level group $(\mathrm{n}=15)$ includes men's badminton players who participate in national competitions and are ranked in the top 100 on the national men's singles badminton ranking list at the moment of the test execution. The nonnational level group $(n=9)$ includes players who take part in regional competitions in the 2018-2019 season and have a ranking exceeding 100 in the national men's singles badminton ranking list at the time of the test. The characteristics of the pro and non-pro groups are presented in Table 1. National players trained significantly more hours per week ( $p<0.001)$ and had higher ratings ( $p<0.001)$ than non-pro players.

A two-fold design was used to evaluate the reproducibility and validity of the test. First, the reproducibility of badminton specific reaction time assessments and inhibition controls was examined using the test-retest design. The time between the initial test and retest ranges from two to three weeks. In the second part of the study, the validity of the four test components is evaluated. The test 
tests its ability to distinguish between national and non-national level players and on the relationship between test results and national rankings.

Badminton Reaction Inhibition Test The newly developed (BRIT) or Badminton Reaction Inhibition Test consists of four components: generaldomain reaction time, badminton-specific reaction time, general-domain inhibition control and badminton-specific inhibition control. Common-domain components are measured by the dominant common stop signal assignment, the procedure is similar to Logan and Cowan's (1984) stop signal assignment. Badminton special components are measured by badminton special stop signal assignments.

Testing is carried out under the supervision of one researcher with the assistance of two other experienced test leaders and takes place during a championship or before a training session. During the testing sessions, the players executed BRIT and filled out questionnaires about badminton-related activities over the past three years, including training hours per week and starting age. Not all players complete all components of the BRIT test and questionnaire due to limited time or reluctance to take physically demanding tests (a special component of BRIT badminton) before a match.

SPSS v.16 was used for data analysis. All test variables were checked for normality by examining the plot of normality, slope z-score and kurtosis. The ranking is considered as ordinal data. For groups of national and non-national level players, the mean and standard deviation of each outcome variable were determined.

Poor specific reaction time reproducibility and inhibition control during go-stop conditions (Badminton Specific Reaction Time of the Go-stop condition, respectively) and gostopBSIC(Badminton Specific Inhibitory Control) was examined by measuring absolute and relative retest reliability and agreement size. Absolute reliability was checked using paired t-test and relative reliability using the intraclass correlation coefficient (ICC, oneway random model). An ICC lower than 0.40 reflects poor reliability, between 0.40 and 0.75 sufficient for good reliability and above 0.75 for excellent reliability (Fleiss, 1999). The size of the agreement is determined by the coefficient of variation (CV) (de Vet et al., 2006).

The construct validity of the four BRIT components was evaluated. This is done by examining the differences between groups (national versus non-national level players) for all test variables separately using one-way analysis of variance, supported by the Cohen effect size. Finally, concurrent validity was investigated by examining the relationship between the national rank and all test variables. This is checked for national and non-national level players separately due to the spread of rankings between the two groups of participants. The Spearman's rho correlation coefficient which is supported by the coefficient of determination as the effect size is calculated for concurrent 
validity checks. Alpha was set at 0.05 for all analyzes.

\section{RESULT}

\section{Reproducibility}

The reproducibility results for poor specific reaction time (BSRT) showed a mean difference between baseline and $1 \mathrm{~ms}$ retest (95\% CI: - 78 - 80) and met the criteria for good relative reliability ( $\mathrm{ICC}=0.626, \mathrm{p}>0,05)$ (Fleiss, 1999). The coefficient of variation (CV) for BSRT is $6 \%$. The badminton-specific inhibitory control (BSIC) showed a mean difference between baseline and retest 31 ms (95\% CI: -47-110) and showed poor to moderate relative reliability (ICC $=0.317$, p> 0.05) ( Fleiss, 1999). The CV for BSIC is $13 \%$.

\section{Construct Validity}

The results of the construct validity analysis are presented in Table 2 . Pro and non-national players did not differ in the domain-general reaction time (DGRT). Pro players outperformed nonpro players on badminton specific reaction time measures during road conditions (Go BSRT) and on badminton specific backhand reaction time measures during road conditions (Go backhand BSRT) ( $\mathrm{p}$ $<0.05$ ). No differences between national and non-national level players were found for badminton specific forehand reaction times during go conditions (Go forehand BSRT) and for domain-general reaction time (DGIC) and Badminton Specific Inhibitory Control (BSIC) (p>0.05)

\section{Concurrent Validity}

The results of the simultaneous validity analysis are presented in Table 3 for pro and non-pro players separately. The national ranking accounts for half of the difference in DGRT'snational and nonnational level players. No significant correlation was revealed between the ratings, BSRT and DGIC. The rating is significantly negatively correlated with BSIC for non-pro players, but not for pro players.

\section{DISCUSSION}

This study evaluates the reproducibility and validity of the newly developed Badminton Reaction Inhibition Test (BRIT) for general and badmintonspecific reaction time assessment and inhibition control in pro and non-pro badminton players. Acceptable reproducibility results for badminton specific reaction times and badminton specific inhibition controls are presented. Good construct validity is shown for badminton specific reaction times because it differentiates between national and nonnational level players. No differences between pro players and national level players were found in general-domain reaction times or in the two inhibition control components. The concurrent validity for general-domain reaction times is good, as it is linked to the national ranking for playerspro and non-pro. No relationship was found between national rankings and badminton specific reaction times or the two inhibitory control components. 
A general-domain stop signal task is used for general-domain cognitive performance appraisal (Logan and Cowan, 1984). The difference between pro and non-pro groups in general-domain reaction time of $10 \mathrm{~ms}$ was not statistically significant. However, in pro badminton, the average shuttle speed is located around $19 \mathrm{~m} / \mathrm{s}$ (Phomsoupha and Laffaye, 2014, 2015), which means that the shuttle moves $19 \mathrm{~cm}$ in $10 \mathrm{~ms}$. This can make the difference between a perfect or bad shuttle return. A significant relationship between general-domain reaction time and national ranking was revealed, indicating the need for fast reaction times for pro performance. This finding underscores the validity of the dominant general stop signal assignment for general reaction time-domain assessments in badminton players.

The reproducibility of the generaldomain stop signal task used for the assessment of general-domain inhibitory control has been confirmed (Congdon et al., 2012; Williams et al., 1999). The current study demonstrates poor relative reliability to suffice for badmintonspecific inhibitory control assessments via badminton-specific stop signal assignments. However, relative reliability is highly dependent on sample size and since the current study includes only five retest measures, future research including a larger sample size is needed to provide an accurate view of the relative reliability of badminton-specific stop signal tasks (Weir, 2005). ). The agreement measure shows a reasonable coefficient of variation of $13 \%$ for badminton-specific obstacle control assessments.
No differences between pro and non-pro players are revealed in badmintonspecific and general-domain inhibition controls. A possible explanation is that BRIT was unable to detect any discrepancies. Another explanation for the finding that pro and non-pro players did not significantly differ in inhibition control could be that the group of participants was too homogeneous. The current study includes pro Dutch badminton players. It can be doubted whether the participants included truly reflect pro badminton performance as the players have ranked higher than 1000 on the World Badminton Federation Men's Singles Ranking. To achieve pro performance in badminton, one must excel in different dimensions (Elferink-Gemser et al., 2011). Perhaps in the current study pro players outperformed non-pros on tactical or physical measures, but not on cognitive performance. However, at the pro level of the badminton world, a player must excel in many domains and compensation from a less developed domain seems impossible. Therefore, future research is recommended to include world-class pro players to provide an accurate evaluation of the need for inhibition control in pro badminton national performance.

BRIT provides an indication of a valid reaction time and can be used by coaches and players to monitor training progress and player progress, it can also provide input for training programs aimed at improving players' badminton performance. Additionally, it can be applied to answer the question of whether highly developed reaction times are innate, causing players with better innate reaction 
skills to have a higher chance of achieving pro badminton performance, or whether pro players have faster reaction times due to fluff. parry them. experience, meaning that faster reaction times can be developed with practice and match play. A review by Smith et al. (2010) who examined the effect of aerobic exercise on neurocognitive performance in healthy adults showed faster reaction times in subjects following aerobic interventions with a duration of more than one month. Although this provides evidence for training reaction times, the literature on the topic is scarce. Future studies should monitor the longitudinal progression of talented badminton players' reaction times to determine whether the progression of reaction times for players who eventually reach pro performance differs from that of non-pro players. Insights into reaction time developments can assist trainers in the process of identifying and developing talent by designing specific training programs. Although this provides evidence for training reaction times, the literature on the topic is scarce. Future studies should monitor the longitudinal progression of talented badminton players' reaction times to determine whether the progression of reaction times for players who eventually achieve pro performance differs from that of non-pro players. Insights into reaction time developments can assist trainers in the process of identifying and developing talent by designing specific training programs. Although this provides evidence for training reaction times, the literature on the topic is scarce. Future studies should monitor the longitudinal progression of talented badminton players' reaction times to find out whether the progression of reaction times for players who eventually reach pro performance differs from that of non-pro players. Insights into reaction time developments can assist trainers in the process of identifying and developing talent by designing specific training programs. Future studies should monitor the longitudinal progression of talented badminton players' reaction times to find out whether the progression of reaction times for players who eventually reach pro performance differs from that of non-pro players. Insights into reaction time developments can assist trainers in the process of identifying and developing talent by designing specific training programs. Future studies should monitor the longitudinal progression of talented badminton players' reaction times to determine whether the progression of reaction times for players who eventually reach pro performance differs from that of non-pro players. Insights into reaction time developments can assist trainers in the process of identifying and developing talent by designing specific training programs.

This study has several limitations. One of them is the small number of participants in the reproductive section of the study. Future research including larger sample sizes and pro world players should be undertaken to gain a better view of reproducibility and validity for inhibitory control assessments. Furthermore, the validity and reproducibility of BRIT were only assessed in the adult population. It is advisable to carry out similar studies on children of different ages and levels of performance to check BRIT scores for monitoring badminton performance in 
different age groups. The current study is focused on evaluating the reproducibility and validity of the newly developed test for badminton-specific and general-domain reaction time assessment as well as inhibition control in badminton players.

In conclusion, the reproducibility and validity of the inhibitory control assessment are not confirmed, however, BRIT appears to be a valid and reproducible measure of reaction time in badminton players. Reaction times as measured by BRIT can provide input for training programs aimed at improving badminton player performance

\section{REFERENCES}

Alves H, Voss MW, Boot WR, Deslandes A, Cossich V, Salles JI, Kramer AF. Perceptual-cognitive expertise in elite volleyball players. Front Psychol, 2013; 4: $1-9$

Congdon E, Mumford JA, Cohen JR, Galvan A, Canli T, Poldrack RA. Measurement and reliability of response inhibition. Front Psychol, 2012; 3: 1-10

Diamond A. The early development of executive functions. Lifespan Cognition: Mechanisms of Change. Oxford University Press, 70-95; 2006

Elferink-Gemser MT, Jordet G, Coelho-ESilva MJ, Visscher C. The marvels of elite sports: how to get there?Brit J Sport Med, 2011; 45: 683-684

Faber IR, Bustin PMJ, Oosterveld FGJ, Elferink-Gemser MT, Nijhuis-Van der Sanden MWG. Assessing personal talent determinants in young racquet sport players: a systematic review. J Sports Sci, 2016; 34: 395-410

Faber IR, Nijhuis-Van Der Sanden MWG, Elferink-Gemser MT, Oosterveld FGJ. The Dutch motor skills assessment as tool for talent development in table tennis: a reproducibility and validity study. J Sports Sci, 2015; 33: 1149-1158
Elferink-Gemser MT, Oosterveld FGJ, Nijhuis-Van der Sanden MWG. Revision of two test items of the dutch motor skills assessment measuring ball control in young table tennis players: a reproducibility and validity study. Ann Res SportPhys Act, 2014: 53-69

Fleiss JL. The Design and Analysis of Clinical Experiments. Hoboken: John Wiley \& Sons, Inc; 1999 Hong Y, Wang SJ, Lam WK, Cheung JTM. Kinetics of badminton lunges in four directions. $\mathrm{J}$ Appl Biomech, 2014; 30: 113-118

Huijgen BCH, Leemhuis S, Kok N, Verburgh, L, Oosterlaan, J, Elferink-Gemser, MT, Visscher, C. Cognitive functions in elite and sub-elite youth soccer players aged 13 to 17 years. PloS One, 2015; 10: e0144580

Kida N, Oda S, Matsumura M. Intensive baseball practice improves the go/nogo reaction time, but not the simple reaction time. Cognitive Brain Res, 2005; 22: 257-264

Kuntze G, Mansfield N, Sellers WA. Biomechanical analysis of common lunge tasks in badminton. J Sport Sci, 2010; 28: 183-191

Lees A. Science and the major racket sports: a review. J Sport Sci, 2003; 21: 707-732

Logan GD, Cowan WB. On the ability to inhibit thought and action: a theory of an act of control. Psychol Rev, 1984; 91: 295-327

Loureiro LFB, Freitas PB. Influence of the performance level of badminton players in neuromoter aspects during a targetpointing task. Rev Bras Med Esporte, 2012; 18: 203-207

Madsen CM, Karlsen A, Nybo L. Novel speed test for evaluation of badminton-specific movements. J Strength Cond Res, 2015; 29: 1203-1210

Mann D, Williams A, Ward P, Janelle C. Perceptual-cognitive expertise in sport: a meta-analysis. J Sport Exercise Psy, 2007; 29: 457-464

Phomsoupha M, Laffaye G. Shuttlecock velocity during a smash stroke in badminton evolves linearly with skill level. Comput Meth Biomechanics and Biomed Eng, 2014; 17: 140-141 
Phomsoupha M, Laffaye G. The science of badminton: game characteristics, anthropometry, physiology, visual fitness and biomechanics. Sports Med, 2015; 45: 473-495

Smith PJ, Blumenthal JA, Hoffman BM, Cooper H, Strauman TA, Welsh-Bohmer K, Browndyke JN, Sherwood A. Aerobic exercise and neurocognitive performance: a meta-analytic review of randomized controlled trials. Psychosom Medicine, 2010; 72: 239-252

Verbruggen F, Logan GD. Models of response inhibition in the stop-signal and stopchange paradigms. Neurosci Biobehav R, 2009; 33: 647-61

Verburgh L, Scherder EJA, van Lange PAM, Oosterlaan J. Executive functioning in highly talented soccer players. PloS One, 2014; 9: e91254

Vestberg T, Gustafson R, Maurex L, Ingvar M, Petrovic P. Executive functions predict the success of topsoccer players. PloS One, 2012; 7: e34731 de Vet HCW, Terwee CB, Knol DL, Bouter LM. When to use agreement versus reliability measures. J Clin

Voss MW, Kramer AF, Basak C, Prakash RS, Roberts B. Are expert athletes "expert" in the cognitive laboratory? A metaanalytic review of cognition and sport expertise. Appl Cognitive Psych, 2010; 24: 812-826

Weir J. Quantifying test-retest reliability using the intraclass correlation coefficient and the SEM. J Strength Cond Res, 2005; 19: 231-240

Wetherell A. Cognitive and psychomotor performance tests and experiment design in multiple chemical sensitivity. Environ Health Persp, 1997; 105: 495-503

Williams BR, Ponesse JS, Schachar RJ, Logan GD, Tannock R. Development of inhibitory control across the life span. Dev Psychol, 1999; 35: 205-213 